\title{
Concentración, morfología y composición química de partículas PST y PM10, colectadas en la chimenea de tres motores de combustión interna de alta capacidad (42 MW) Concentration, morphology and chemical composition of TSP and PM10 collected in the stack of three internal combustion engines of high capacity (42 MW)
}

\author{
Martínez-Flores Marco Antonio \\ Instituto Nacional de Electricidad y Energías Limpias \\ División Energías Alternas \\ Correo: mamf@iie.org.mx \\ Tamayo-Flores Gustavo Adolfo \\ Instituto Nacional de Electricidad y Energías Limpias \\ División Energías Alternas \\ Correo: gatamayo@iie.org.mx \\ Barajas-Martínez Dulce Karime \\ Universidad Politécnica del Estado de Morelos \\ Ingeniería Ambiental \\ Correo: karimebm221@gmail.com \\ Hernández-Flores Nicasio \\ Instituto Nacional de Electricidad y Energías Limpias \\ División Energías Alternas \\ Correo: Nicasio.hernandez@iie.org.mx
}

Celada-Murillo Ana Teresa

Instituto Nacional de Electricidad y Energías Limpias

División Energías Alternas

Correo: atcelada@iie.org.mx

Arias-Osorio Alfonso
Comisión Federal de Electricidad
Subdirección de Construcción
Departamento de Protección Ambiental
Correo: alfonso.arias@cfe.gob.mx
Cortes-López Elizabeth
Comisión Federal de Electricidad
Subdirección de Construcción
Departamento de Protección Ambiental
Correo: elizabeth.cortes@cfe.gob.mx
Colin-Aguilar Ana Laura
Instituto Nacional de Electricidad y Energías Limpias
División Energías Alternas
Correo: Ana.laura.colin@iie.org.mx

\section{Resumen}

En este estudio se presentan los primeros resultados en México de la concentración, morfología, y composición química de partículas suspendidas totales (PST) y partículas con diámetros aerodinámicos menores de 10 micras (PM10) emitidas por tres motores de combustión interna $(\mathrm{MCl})$ de 42 megawatts $(\mathrm{MW})$, empleados para generar energía eléctrica. La campaña se realizó en noviembre de 2015 y el muestreo se efectuó siguiendo los métodos 5 y 201A EPA. El combustible utilizado en los MCl-2 y MCl-3, fue un combustóleo mexicano con 4\% de azufre, y gravedad específica mayor que 1. El MCl-1 utilizó una mezcla con 85\% de combustóleo y 15\% diésel. La concentración de PST en el MCl-1 fue de $178 \mathrm{mg} / \mathrm{Nm}^{3}, 190 \mathrm{mg} / \mathrm{Nm}^{3}$ en el MCl-2 y $151 \mathrm{mg} / \mathrm{Nm}^{3}$ en el MCl-3. El porcentaje en peso de las PM10 en relación con las PST fue de 62\% en el MCl-1 y de 80\% en los MCl-2 y MCl-3. La morfología de las PST y las PM10 en cada $\mathrm{MCl}$ se comparó contra la de las partículas de un horno experimental de $0.35 \mathrm{MW}$. Las partículas emitidas por los $\mathrm{MCl}$ fueron sólidas, esféricas, con tamaños menores de 10 micras, difiriendo de las PST del horno piloto, las cuales son amorfas, porosas y de diversos tamaños. La composición química de las partículas de los tres $\mathrm{MCl}$ tuvo como componentes principales al O, C, V y S.

Descriptores: PST, PM10, emisiones, morfología, motores combustión interna.

\begin{abstract}
The first Mexican results of the concentration, morphology and chemical composition of Total Suspended Particles (TSP) and particles smaller than 10 microns (PM10) from three Internal Combustion Engines (ICE) of $42 \mathrm{MW}$, employees to generate electricity, which were obtained by an experimental campaign on November, 2015 and follows the sampling procedures described in the EPA methods 5 and 201A. The ICE's used two kind of fuels: in the ICE-2 and ICE-3, it was a Mexican heavy oil with $4 \%$ by weight of sulfur, $0.07 \%$ ash and a specific gravity greater than 1; while in ICE-1, it was used a mixture with $85 \%$ of Mexican Heavy oil and $15 \%$ of diesel. The TSP concentration in ICE-1 was of $178 \mathrm{mg} / \mathrm{Nm} 3$ in which $62 \%$ were PM10; for the case of the ICE-2 and ICE-3, the TSP were of 190 and $151 \mathrm{mg} / \mathrm{Nm} 3$, respectively, and in both cases the PM10 were the $80 \%$ of the total TSP. The morphology of the TSP and PM10 in all ICE's, were of solid and spherical particles, with the majority of the sizes under $10 \mu \mathrm{m}$. When this morphology is compared with the TSP of a conventional furnace, the amorphous, porosity and the wide variety of sizes are the main differences. As principal components in the particles of the ICE's were identified the $\mathrm{O}, \mathrm{C}, \mathrm{V}$ and $\mathrm{S}$.
\end{abstract}

Keywords: TSP, PM10, emission, morphology, internal combustion engines. 


\section{INTRODUCCIÓN}

El tamaño y composición del material particulado (PM) son dos de los parámetros más importantes en la determinación del impacto que estos producen sobre la salud humana y el medio ambiente. Una forma de clasificar el tamaño de las partículas se basa en su diámetro aerodinámico, este indicador es igual al diámetro de una partícula esférica de densidad unitaria que tiene la misma velocidad terminal que la partícula considera$\mathrm{da}$, independiente de su forma, tamaño o densidad bajo condiciones de temperatura, presión y humedad existentes (World Health Organization Europe, 2005), y en esta clasificación se tiene que las PST son aquellas que comprenden un rango de tamaño entre 0.005 y $100 \mu \mathrm{m}$ (EPA,1996; INE, 2010), por su parte, las partículas PM10 son aquellas cuyo diámetro aerodinámico es menor o igual a $10 \mu \mathrm{m}$.

Las PM10 se encuentran asociadas con fuentes de emisión antropogénicas y forman parte de la fracción respirable, por lo que su potencial de afectación a seres vivos es mayor al de las PST. Las partículas antropogénicas muestran diversos niveles de oxidación, que dependen principalmente del proceso por el que fueron emitidas. Aquellas partículas que provienen de procesos de combustión de calderas industriales, se caracterizan por contar con una figura no uniforme y hueca (también conocidas como cenósferas). Las PM10, consisten de una mezcla compleja de compuestos de naturaleza orgánica e inorgánica, con diferentes distribuciones granulométricas, las cuales están condicionadas por la composición de los gases que las rodean (Querol, 2005). El mecanismo de formación, así como la fuente de emisión de las partículas son factores que influyen directamente en la composición y características de las mismas, dando como resultado que las PM10 muestren una gran variedad de formas: esferas, elipses, cubos, formas irregulares o geometría fractal. En el caso de su composición química, las PM10 pueden estar constituidas por metales como silicio, calcio, zinc, plomo, hierro y cadmio, así como por compuestos de nitrógeno, azufre y carbono (Garrido y Camargo, 2012).

Es importante señalar, que en México no se tienen antecedentes sobre la medición de PM10 en motores de combustión interna de gran capacidad (p. ej. los utilizados para la producción de energía eléctrica comercial) $\mathrm{y}$, por tanto, tampoco sobre su tasa de emisión, morfología y composición química. El proceso que se lleva a cabo en un motor de combustión interna y la información de sus características de atomización son poco conocidas. En los motores de combustión interna se emplean "Inyectores" para la atomización del combustible. El tiempo con que se cuenta para llevar a cabo su oxidación y liberación de calor es de fracciones de segundo comparado con el tiempo que se tiene en el hogar de un generador de vapor de gran capacidad.

El objetivo de este estudio fue determinar la concentración, morfología y composición química de las PST y PM10 emitidas por tres motores de combustión interna, de $42 \mathrm{MW}$ cada uno. Lo anterior permitirá realizar una comparación entre ambas emisiones (concentración, morfología y composición química), y observar si los datos sobre PST y PM10 están en cumplimiento del marco legar regulado.

\section{Desarrollo}

La campaña experimental tuvo una duración de 10 días, en el periodo del 10 al 19 de noviembre de 2015, y en ella se colectaron muestras de las partículas que se emiten a la atmósfera por las chimeneas de tres MCI de alta capacidad de combustión (42 MW).

Tabla 1. Características principales de las $\mathrm{MCl}$

\begin{tabular}{cccc}
\hline Característica & \multicolumn{3}{c}{ Motor } \\
\hline Máquina de combustión interna & MCI-1 & MCI-2 & MCI-3 \\
Año de construcción & 2003 & 2011 & 2012 \\
Capacidad nominal $(\mathrm{kW})$ & 43,950 & 42,840 & 42,840 \\
Combustión interna & Dos tiempos & Dos tiempos & Dos tiempos \\
Cilindros & 10 & 12 & 12 \\
Diámetro de cilindro $(\mathrm{cm})$ & 90 & 80 & 230 \\
Carrera del cilindro $(\mathrm{cm})$ & 230 & 230 & 109.2 \\
Velocidad nominal $(\mathrm{rpm})$ & 109.2 & 109.2 & Combustóleo \\
\hline
\end{tabular}




\section{CARACTERÍSTICAS DEL EQUIPO DE COMBUSTIÓN}

Las máquinas de combustión interna donde se realizaron las pruebas experimentales, son de baja velocidad, con combustión de dos tiempos y de ignición por compresión. En la Tabla 1, se presentan las características principales de las MCI.

\section{MÉTODOS DE MEDICIÓN}

En la chimenea de cada máquina de combustión interna, se realizó una medición de las PST y una de las PM10, así como del exceso de oxígeno $\left(\mathrm{O}_{2}\right)$ y la temperatura y presión dinámica y estática de los gases de combustión. Las últimas tres variables se utilizaron para determinar la velocidad y el flujo de gases de combustión que son expulsados a la atmosfera a través de la chimenea.

Tal como se indicó anteriormente, la metodología de medición de PST y PM10 estuvo basada en los métodos 1 (1994), 2 (2011), 3 (2009), 4 (2009), 5 (2009) y 201A (2010) de la EPA, mismos que se describen a continuación.

\section{Método 1. Determinación de puntos de MUESTREO}

Está diseñado para realizar una medición representativa de las emisiones contaminantes o del flujo volumétrico de una fuente estacionaria y debe aplicarse en ductos con diámetros iguales o mayores a $30 \mathrm{~cm}$ y donde el flujo no sea ciclónico o con remolinos. Además, establece que el sitio de medición debe encontrarse a una distancia mínima de 8 diámetros internos del ducto o chimenea después de cualquier perturbación y 2 diámetros antes de la siguiente, y bajo estas condiciones el mínimo número de puntos de medición será de 12 .

En el caso de esta investigación, la altura de la chimenea de los tres MCI fue de $70 \mathrm{~m}$ con un diámetro in- terno de $3 \mathrm{~m}$. Por otro lado, los sitios de medición en los MCI-2 y 3, se ubicaron a una altura de 40 m ( $\approx 13.3$ diámetros) respecto a la perturbación aguas abajo y $30 \mathrm{~m}$ (10 diámetros) en relación con la perturbación aguas arriba. Mientras que, para el MCI-1 la zona de medición se localizó a 60 m (20 diámetros) y 10 m ( $\approx 3.3$ diámetros) con referencia a la perturbación aguas abajo y aguas arriba, respectivamente. Estas condiciones dan un cabal cumplimiento de los términos indicados en el Método 1 y con base en esto, el número mínimo puntos de medición fue de 12, distribuidos como se muestra en la Figura 1.

\section{MÉtodo 2. DeterminaCión de la VelOCIDAD DEL GAS Y FLUJO VOLUMÉTRICO EN LA CHIMENEA}

Es aplicable para medir la velocidad promedio y para cuantificar el flujo volumétrico o caudal del gas. La velocidad y el flujo volumétrico del gas en la chimenea se determinan a través de la densidad del gas y de la medición de la presión de velocidad promedio (presión dinámica) con un tubo de pitot tipo " $\mathrm{S}$ ". En esta investigación, se aplicó este método debido a que los sitios de medición cumplen con los requisitos indicados en el Método 1.

El flujo de un gas $\left(W_{f}\right)$ a través de un conducto, es función directa de la velocidad promedio del gas $\left(\mathrm{V}_{\mathrm{s}}\right)$ y del área de la sección transversal del conducto (A).

$W_{f}=V_{s} \times A$

Donde la velocidad es función directa de la presión dinámica $(\Delta P)$ y la densidad del fluido $(\rho)$.

$$
V_{s}=K \sqrt{\frac{2 \times \Delta P}{\rho}}
$$

O bien, se calcula mediante la siguiente expresión:

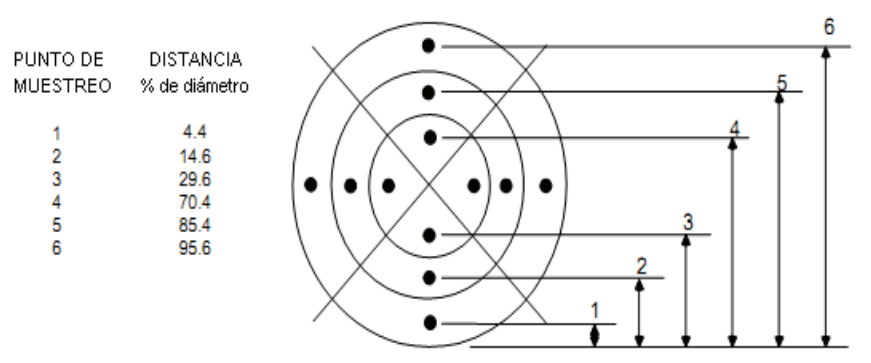

Ejemplo de sección transversal de chimenea circular dividida en 12 áreas iguales, indicando la localización de los puntos de muestreo.

\begin{tabular}{ccc}
\hline $\begin{array}{c}\text { Punto de } \\
\text { muestreo }\end{array}$ & $\begin{array}{c}\text { Distancia } \\
(\%)\end{array}$ & $\begin{array}{c}\text { Distancia } \\
(\mathrm{cm})\end{array}$ \\
\hline 1 & 4.4 & 13.2 \\
2 & 14.6 & 43.8 \\
3 & 29.6 & 88.8 \\
4 & 70.4 & 211.2 \\
5 & 85.4 & 256.2 \\
6 & 95.6 & 286.8 \\
\hline
\end{tabular}

Diámetro de la chimenea $300 \mathrm{~cm}$

Figura 1. Distribución de puntos de medición en el área de la chimenea de cada $\mathrm{MCI}$ 
$V_{s}=K p \times F c \sqrt{\frac{T c h}{P M \times P S}(\Delta P)^{1 / 2}}$

$P s=P b+P e$

donde:

$V_{s}=$ Velocidad de los gases en la chimenea, $\mathrm{m} / \mathrm{s}$

$K p=$ Constante del tubo de Pitot, adimensional

$F c=$ Factor de calibración del tubo de Pitot, adimensional

$T c h=$ Temperatura de gases en chimenea, $\mathrm{K}$

$P M=$ Peso molecular de gases en chimenea en base húmeda, g/gmol

$\Delta P^{1 / 2}=$ Presión dinámica de gases en la chimenea, $\mathrm{mm}$ $\mathrm{H} 2 \mathrm{O}$

Ps = Presión absoluta de gases en la chimenea, $\mathrm{mm}$ $\mathrm{Hg}$

$\mathrm{Pb}=$ Presión barométrica del sitio de medición, $\mathrm{mm}$ $\mathrm{Hg}$

$\mathrm{Pe}=$ Presión estática de los gases en chimenea, $\mathrm{mm}$ $\mathrm{H}_{2} \mathrm{O}$

\section{Método 3. Análisis de Gases para la determinación} DE PESO MOLECULAR DE LOS GASES SECOS

Se aplica para determinar las concentraciones de monóxido de carbono (CO), bióxido de carbono $\left(\mathrm{CO}_{2}\right)$ oxígeno $\left(\mathrm{O}_{2}\right)$, aire en exceso y peso molecular del gas seco (Md) de una muestra de gases de combustión de un combustible fósil. La determinación del peso molecular del gas seco, se realizó mediante el empleo de un analizador automático con sensores electroquímicos, los cuales, determinan la concentración del contaminante en partes por millón (ppm) o porciento volumen $(\mathrm{cmol} /$ mol): $\mathrm{O}_{2}, \mathrm{CO}$ y calculan la concentración de $\mathrm{CO}_{2}$. Una vez determinada la concentración de las especies señaladas, se determina el peso molecular de los gases, mediante la siguiente expresión:

$\mathrm{Md}=0.44\left(\% \mathrm{CO}_{2}\right)+0.32\left(\% \mathrm{O}_{2}\right)+0.28\left(\% \mathrm{CO}+\% \mathrm{~N}_{2}\right)$

$\% \mathrm{~N}_{2}=100-\% \mathrm{CO}_{2}-\% \mathrm{O}_{2}-\% \mathrm{CO}$

donde:

$M d=$ Peso molecular del gas seco, $\mathrm{kg} / \mathrm{Kmol}$

$\% \mathrm{~N}_{2}=$ Concentración de nitrógeno en base seca, $\mathrm{cmol} / \mathrm{mol}$

$\% \mathrm{CO}_{2}=$ Concentración de bióxido de carbono en base seca, $\mathrm{cmol} / \mathrm{mol}$
$\% \mathrm{O}_{2}=$ Concentración de oxígeno en base seca, cmol/ mol

$\% \mathrm{CO}=$ Concentración de monóxido de carbono en base seca, $\mathrm{cmol} / \mathrm{mol}$

\section{MÉTOdO 4. MUESTREO PARA DETERMINACIÓN}

DE HUMEDAD EN CHIMENEA

Con él se cuantifica el contenido de humedad de los gases de la chimenea y describe dos procedimientos: El primero es un método de referencia, para determinaciones exactas del contenido de humedad (tal como son necesarias para calcular tasas de emisión). El segundo es un método aproximado que suministra estimativos del porcentaje de humedad para ayudar en el establecimiento del muestreo isocinético con anterioridad a la medición de la emisión de contaminantes. En esta investigación se aplicaron los dos métodos.

Los cálculos de humedad incluyen:

$V_{m s t}=K_{1} \times Y \times \frac{V m \times\left(P b+\frac{\Delta H}{13.6}\right)}{T_{m}}$

donde

$V_{\text {mst }}=$ Volumen de gas seco medido por el medidor de gas seco, corregido en condiciones estándar $\left(20^{\circ} \mathrm{C}, 760 \mathrm{~mm} \mathrm{Hg}\right), \mathrm{m}^{3}{ }_{\text {std }}$

$K_{1}=0.3858 \mathrm{~K} / \mathrm{mm} \mathrm{Hg}$

$Y=$ Constante de calibración del medidor de gas seco (orificio), adimensional

$V m=$ Volumen medido por el medidor de gas seco, $\mathrm{m}^{3}$

$\mathrm{Pb}=$ Presión barométrica del sitio de medición, $\mathrm{mm} \mathrm{Hg}$

$\Delta \mathrm{H}=$ Presión de diferencial promedio a través del orificio de medición, $\mathrm{mm} \mathrm{H}_{2} \mathrm{O}$

$T_{m}=$ Temperatura del medidor de gas seco, $\mathrm{K}$

$V_{w s t}=K_{2} \times\left(W_{T}\right)$

$B_{w s}=\left(\frac{V_{w s t}}{V_{w s t}+V_{m s t}}\right) \times 100=\%$

$M s=M_{d} \times\left(1-B_{w s}\right)+18 \times B_{w s}$

donde

$V_{\text {wst }}=$ Volumen de vapor de agua colectada en los impactores a condiciones estándar, $\mathrm{m}_{\text {std }}^{3}$ 
$K_{2}=0,001335 \mathrm{~m}^{3} / \mathrm{ml}$

$W_{T}=$ Volumen de agua recolectada en los impacto res a condiciones de medición, $\mathrm{ml}$ o $\mathrm{g}$

$B_{w s}=$ Proporción de vapor de agua en volumen en la corriente de gas, $\%$

$M s=$ Peso molecular del gas húmedo, en $\mathrm{kg} / \mathrm{kg}-\mathrm{mol}$

\section{Método 5: Muestreo para Determinación de Material \\ PARTICULADO}

Se aplica para la determinación de las emisiones de masa de partículas de fuentes estacionarias. Las partículas se remueven isocinéticamente de la fuente y se recolectan sobre un filtro de fibra de vidrio, mantenido a una temperatura en el rango de $120 \pm 14^{\circ} \mathrm{C}$. La masa de las partículas, que incluye cualquier material que se condense a, o por encima de la temperatura de filtración, se determina gravimétricamente después de la eliminación del agua no combinada. En la Figura 2, se muestra un diagrama esquemático del equipo de medición.

En este método se emplean los cálculos y determinaciones de los métodos 1 a 4 y se calcula el diámetro de la boquilla y el factor de Isocineticidad para validar las mediciones realizadas, utilizando las formulas siguientes:

$\phi_{n}=\sqrt{\frac{0.6071 \times Q_{m} \times P_{m} \times\left(1-B_{w m}\right)}{T_{m} \times C_{p} \times\left(1-B_{w s}\right)} \sqrt{\frac{T_{s} \times M_{s}}{P_{s} \times \Delta P}}}$

donde

$\varnothing n$ = Diámetro interno de la boquilla de la sonda, $\mathrm{mm}$
$\mathrm{Qm}=$ Constante de calibración del medidor de orificio, 2.38 adimensional

$C p=$ Coeficiente del Pitot, adimensional, 0.85 para el pitot usado en este trabajo

$B w m=$ Porcentaje humedad presente en medidor gas seco, $\%$, se asume $=0.0$

$T_{s}=$ Temperatura de los gases en chimenea, $\mathrm{K}$

$\Delta P=$ Presión dinámica de la corriente de gases en chimenea, $\mathrm{mm} \mathrm{H}_{2} \mathrm{O}$

$M s$ = Peso molecular del gas húmedo de la chimenea, en $\mathrm{kg} / \mathrm{kg}$-mol

$B_{w s}=$ Proporción de vapor de agua, en volumen, en la corriente de gas, \%vol

El factor de Isocineticidad se determina de la siguiente forma:

$I=\frac{100 T_{s}\left(K_{4} V_{1 c}+\frac{\left(V_{m} Y\right)}{T_{m}}\left(P_{b}+\frac{\Delta H}{13.6}\right)\right)}{60 \theta V_{s} P_{s} A_{n}}$

donde

$\begin{array}{ll}K_{4}= & 0.003454 \frac{\mathrm{mmHg} \mathrm{m}}{\mathrm{ml} K} \\ V_{1 c}= & \begin{array}{l}\text { Volumen total del líquido colectado en los im- } \\ \text { pactores y sílica gel, } \mathrm{ml}\end{array} \\ \theta= & \begin{array}{l}\text { Tiempo total de medición, min } \\ A_{n}=\end{array} \quad \begin{array}{l}\text { Área de la sección transversal de la boquilla, } \\ \mathrm{mm}^{2}\end{array}\end{array}$

La emisión de partículas $\mathrm{C}_{\mathrm{s}^{\prime}}$ se determina mediante la siguiente ecuación:

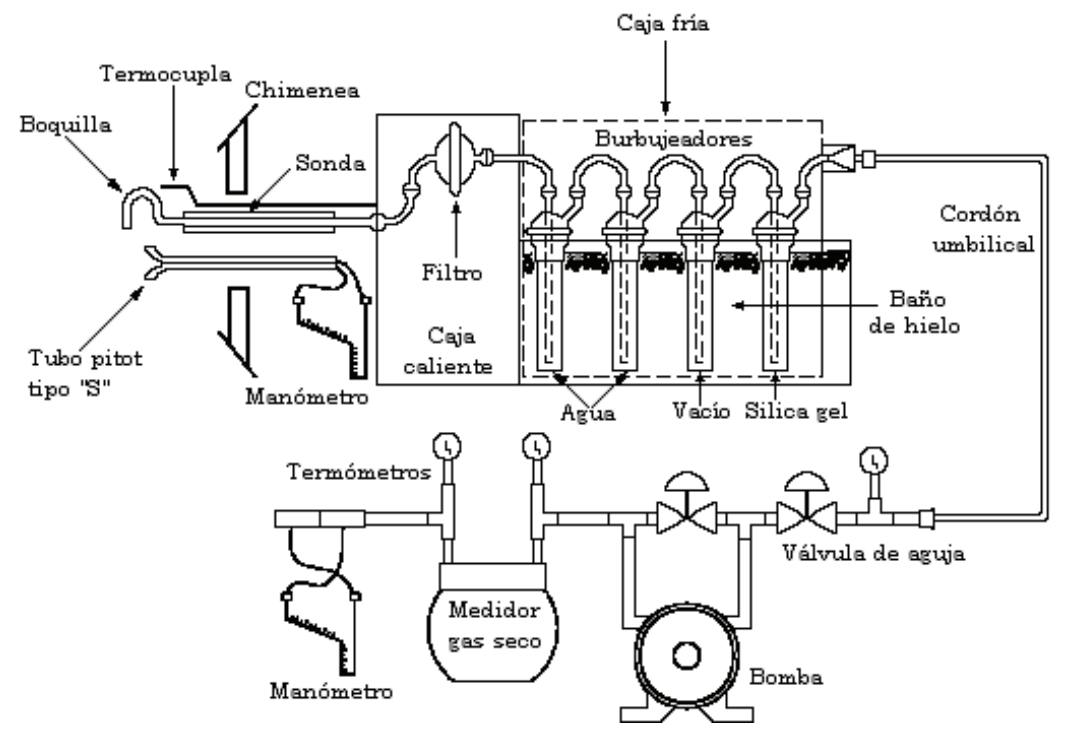

Figura 2. Tren de muestreo, método 5 de la EPA 
$C_{s}=\frac{K_{3} m_{n}}{V_{m s t}}$

donde

$m_{n=} \quad$ Cantidad total de partículas colectadas, $\mathrm{mg}$

$\mathrm{K}_{3}=\quad 0.001 \mathrm{~g} / \mathrm{mg}$

Método 201A. Medición de PARTí́CULAS CON DiÁMETro AERODINÁMICO MENOR O IGUAL A 10 MICRÓMETROS EN FUENTES FIJAS ESTACIONARIAS

En la Figura 3, se presenta un diagrama del ciclón I con diámetro de corte de $10 \mu \mathrm{m}$, las dimensiones de este tipo de ciclones se muestran en la Tabla 2. Este dispositivo es capaz de separar las partículas con diámetros aerodinámicos mayores que $10 \mu \mathrm{m}$, teniendo como condicionante que el muestreo se realice en un rango de 80 a 120 por ciento del flujo isocinético. La muestra de gas se debe extraer de la chimenea a una razón constante de flujo.

En esta investigación se utilizó un ciclón que cumple con las especificaciones recomendadas por el método 201A.

Las tasas de emisión reales de PST y PM10 de cada equipo de combustión en observación, se determinan mediante la siguiente expresión:

$\dot{m}_{i}=C i^{*} W_{f}$ donde

$\dot{m}_{i}=$ Tasa de emisión del componente i de los gases de escape a condiciones de medición, $\mathrm{g} / \mathrm{s}$

$C_{i}=$ Concentración en masa del componente i de los gases de escape a condiciones de medición, $\mathrm{mg} / \mathrm{m}^{3}$

$W_{f}=$ Flujo volumétrico de los gases de escape húmedos, $\mathrm{m}^{3} / \mathrm{s}$

DETERMINACIÓN DE LA MORFOLOGÍA Y COMPOSICIÓN QUÍMICA ELEMENTAL DE LAS PARTÍCULAS

La morfología y composición química elemental de las partículas obtenidas en los MCI-1, MC-2 y MC-3, se determinó con un microscopio electrónico de barrido (SEM).

El SEM utilizado cuenta con un espectrómetro dispersivo de energía, el cual produce un haz de electrones con un alto voltaje de aceleración que incide sobre la muestra de partículas. Su diseño consta de detectores de electrones secundarios (SE) con una resolución de $3 \mathrm{~nm}$ que proporcionan imágenes de la superficie topográfica de la muestra, detectores de electrones retrodispersados (BSE) con una resolución de $4 \mathrm{~nm}$ que proporcionan imágenes en escala de grises referentes a los elementos constituyentes de la muestra y un detector de energía de rayos X (EDS) capaz de realizar la medición semi-cuantitativa de los elementos presentes en la muestra.

Tabla 2. Especificaciones de diseño para el ciclón I (10 $\mu \mathrm{m})$

\begin{tabular}{ccccccccccccc}
\hline & \multicolumn{10}{c}{ Dimensiones interiores del ciclón $(\mathrm{cm} \pm 0.02 \mathrm{~cm})$} \\
Ciclón I $(10$ & Din & $\mathrm{D}$ & $\mathrm{De}$ & $\mathrm{B}$ & $\mathrm{H}$ & $\mathrm{h}$ & $\mathrm{Z}$ & $\mathrm{S}$ & Hcup & Dcup & De $^{\prime}$ & Do \\
$\mu \mathrm{m})$ & 1.27 & 4.47 & 1.5 & 1.86 & 6.95 & 2.24 & 4.71 & 1.57 & 2.25 & 4.45 & 1.02 & 1.24 \\
\hline
\end{tabular}

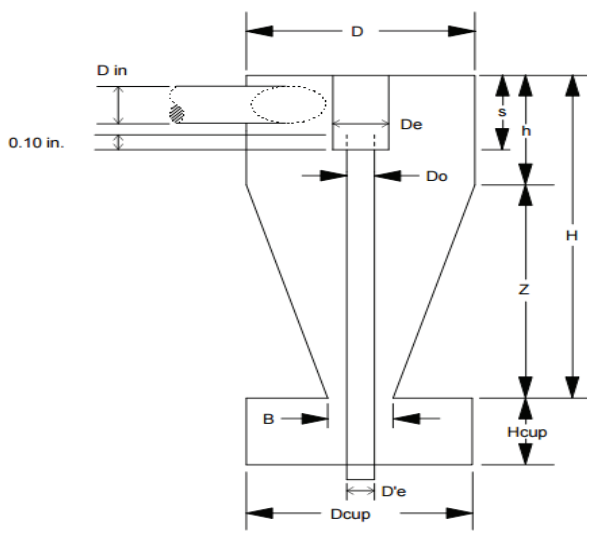

Figura 3. Diagrama del ciclón I para partículas PM10 


\section{DisCuSIÓN Y ANÁLISIS DE RESULTADOS}

\section{Propiedades del combustible}

Durante las pruebas experimentales, el MCI-1 trabajó con una mezcla de $85 \%$ combustóleo y $15 \%$ de diésel (esta condición operativa se recomendó por el fabricante durante las pruebas de desempeño del MCI-1), mientras que los MCI-2 y 3 utilizaron solo combustóleo. En la Tabla 3 se muestran las principales propiedades de ambos combustibles, y como se observa, la mezcla de combustible utilizada por el MCI-1, tiene pequeñas diferencias en todas sus propiedades comparado con el combustóleo utilizado en los MCI-2 y 3. Por ejemplo, las propiedades de temperatura de inflamación y gravedad específica nos indican que ambos son combustibles pesados y por ende difíciles de quemar. Por último, el contenido de azufre nos sugiere que los MCI-2 y 3 emitirán una mayor cantidad de productos de azufre $\left(\mathrm{SO}_{\mathrm{x}}\right)$ a la atmósfera.

\section{CONDICIONES EXPERIMENTALES}

En la Tabla 4, se muestran las condiciones generales de los gases en chimenea bajo las cuales se realizaron las pruebas experimentales de medición de PST y PM10. Tal como se observa, el MCI-1 operó a una carga menor que los MCI-2 y 3, pese a ello, la velocidad de los gases fue mayor, pues esta varía directa y proporcionalmente con la raíz cuadrada de la temperatura, la cual, también fue la más elevada. En contraste, el flujo másico (que no es dependiente de la temperatura) del MCI-1 fue menor, ya que, al trabajar a una carga más baja requirió menos combustible y aire y por consiguiente expulsó menos masa de gases.

\section{CONCENTRACIÓN DE PARTíCULAS}

El factor de isocineticidad durante la medición de partículas es un parámetro muy importante en la aplicación de los métodos 5 y 201A. La isocineticidad significa que la muestra de gases se extrae a la misma velocidad con que viajan los gases de combustión en el ducto en que se muestrea, de este modo, se evita la separación o selección de partículas por diferencia de tamaño o peso específico (Espinoza, 1990). Para asegurar lo anterior, en el método 5 se establece un factor de isocineticidad de $100 \pm 10 \%$, para el método $201 \mathrm{~A}$ se establece que el factor isocinético debe ser $100 \pm 20 \%$. De la Tabla 5, se puede observar que para las pruebas experimentales realizadas el factor isocinético cae dentro del rango especificado por los métodos 5 y 201A.

En relación con las normas de emisión vigentes, los motores de combustión interna no están considerados por la norma oficial mexicana NOM-085-SEMARNAT-2011 para su regulación, sin embargo, la autoridad ambiental acepta que la emisión de PST de estos equipos se compare contra el límite máximo permitido

Tabla 3. Propiedades de los combustibles utilizados en este estudio

\begin{tabular}{cccc}
\hline Propiedad & Unidades & MCI-1 & MCI-2,3 \\
Mezcla 85/15 & Combustóleo \\
\hline Carbono & \% peso & 85.56 & 85.46 \\
Hidrógeno & \% peso & 10.55 & 10.15 \\
Nitrógeno & \% peso & 0.24 & 0.23 \\
Azufre & \% peso & 3.54 & 4.05 \\
Cenizas & $\%$ peso & 0.07 & 0.07 \\
Oxígeno & \% peso & 0.01 & 0.02 \\
Densidad relativa 15.5/15.5 & & 0.9832 & 1.0112 \\
Temperatura de inflamación & \% & 82.5 & 94.5 \\
Agua por destilación & \% Vol & 0.03 & 0.02 \\
Poder calorífico superior & kcal/kg & 10,081 & 9,989 \\
\hline
\end{tabular}


DOI. http://dx.doi.org/10.22201/fi.25940732e.2018.19n3.025

CONCENTRACIÓN, MORFología y COMPOSICIÓN QUíMICA DE PARTíCULAS PST y PM10, COLECTADAS EN LA CHIMENEA DE TRES MOTORES DE COMBUSTIÓN ...

Tabla 4. Condiciones generales de los gases en chimenea

\begin{tabular}{cccccccc}
\hline \multirow{2}{*}{ Variable } & \multirow{2}{*}{ Unidades } & \multicolumn{2}{c}{ MCI-1 } & \multicolumn{2}{c}{ MCI-2 } & \multicolumn{2}{c}{ MCI-3 } \\
\cline { 3 - 8 } & & PST & PM10 & PST & PM10 & PST & PM10 \\
\hline Carga & MW & 26 & 26 & 35 & 38 & 38 & 38 \\
$\mathrm{O} 2$ & $\mathrm{~mol} \%$ & 15.41 & 15.61 & 14.05 & 14.11 & 14.34 & 14.37 \\
$\mathrm{H}$ & $\mathrm{mol} \%$ & 4.6 & 4.8 & 5.6 & 4.0 & 5.4 & 5.0 \\
$\mathrm{Vg}$ & $\mathrm{m} / \mathrm{s}$ & 18.2 & 18.9 & 17.1 & 17.4 & 17.3 & 17.5 \\
$\mathrm{Tg}$ & ${ }^{\circ} \mathrm{C}$ & 278 & 267 & 206 & 207 & 218 & 220 \\
$\mathrm{Qg}$ & $\mathrm{m} / \mathrm{s}$ & 128.6 & 133.6 & 120.9 & 123.0 & 122.3 & 123.7 \\
$\dot{\mathrm{Mg}}$ & $\mathrm{kg} / \mathrm{s}$ & 81.0 & 85.4 & 87.2 & 89.1 & 86.2 & 86.8 \\
$\mathrm{Pe}$ & $\mathrm{mm} \mathrm{H} \mathrm{O}$ & -10.2 & -13.7 & -20.7 & -21.2 & -23.2 & -23.2 \\
$\mathrm{~Pb}$ & $\mathrm{~mm} \mathrm{Hg}$ & 752.5 & 749.8 & 750.1 & 750.3 & 751.0 & 749.9 \\
\hline
\end{tabular}

donde

$\mathrm{O}_{2} \quad$ Exceso de oxígeno en gases de combustión

$\mathrm{H} \quad=$ Humedad en gases medida

$\mathrm{Vg} \quad=$ Velocidad de gases medida

$\operatorname{Tg}=$ Temperatura de gases medida

Qg = Flujo volumétrico de gases húmedos

$\dot{M g}$ = Flujo másico de gases húmedos

Pe $\quad=$ Presión estática de gases

$\mathrm{Pb}=$ Presión barométrica

Tabla 5. Concentración de partículas

\begin{tabular}{cccccccc}
\hline \multirow{2}{*}{ Variable } & \multirow{2}{*}{ Unidades } & \multicolumn{2}{c}{ MCI-1 } & \multicolumn{2}{c}{ MCI-2 } & \multicolumn{2}{c}{ MCI-3 } \\
\cline { 3 - 7 } & & PST & PM10 & PST & PM10 & PST & PM10 \\
\hline Cs & $\mathrm{mg} / \mathrm{Nm}^{3}$ & 66 & 41 & 81 & 66 & 64 & 51 \\
$\mathrm{C}_{\text {scnm }}$ & $\mathrm{mg} / \mathrm{Nm}^{3}$ & 178 & 110 & 190 & 152 & 151 & 121 \\
$\mathrm{C}_{\text {scbm }}$ & $\mathrm{mg} / \mathrm{Nm}^{3}$ & 71 & 46 & 70 & 57 & 58 & 46 \\
$\mathrm{CBM}_{\text {Nmaxemi }}$ & $\mathrm{mg} / \mathrm{Nm}^{3}$ & 50 & --- & 50 & --- & 50 & --- \\
$\dot{m}$ & $\mathrm{~g} / \mathrm{s}$ & 4.3 & 2.8 & 5.7 & 4.8 & 4.4 & 3.6 \\
$\mathrm{I}$ & $\%$ & 90 & 84 & 101 & 104 & 97 & 101 \\
$\mathrm{R}$ & $\%$ & & 61.8 & & 80.0 & & 80.1 \\
\hline
\end{tabular}

donde

Cs = Concentración de partículas en base seca @ $25^{\circ} \mathrm{C}, 1$ atm y $\mathrm{O}_{2}$ medido

$\mathrm{C}_{\mathrm{scnm}}=$ Concentración de partículas en base seca @ $25^{\circ} \mathrm{C}, 1 \mathrm{~atm}$ y $5.0 \% \mathrm{~mol}$ de $\mathrm{O}_{2}$ de referencia

$\mathrm{C}_{\mathrm{scbm}}=$ Concentración de partículas en base seca @ $25^{\circ} \mathrm{C}, 1 \mathrm{~atm}$ y $15.0 \% \mathrm{~mol}$ de $\mathrm{O}_{2}$ de referencia

$\mathrm{CBM}_{\text {Nmaxemi }}=$ Concentración establecida por el GBM de PST en base seca @ $25{ }^{\circ} \mathrm{C}, 1 \mathrm{~atm}$ y $15.0 \%$ mol de $\mathrm{O}_{2}$ de referencia

$\dot{m}=$ Tasa de emisión por unidad de tiempo, g/s

I =Factor isocinético, \%

$\mathrm{R}=$ Relación entre la concentración PM10/PST utilizando los valores corregidos $\left(\mathrm{C}_{\mathrm{scnm}}\right), \%$ 
por la norma referida para equipos con una capacidad de carga mayor a $28 \mathrm{MW}$, pero menor a $147 \mathrm{MW}$. En este caso, la NOM-085-SEMARNAT-2011 establece un nivel máximo permisible de emisión de PST de $350 \mathrm{mg} /$ $\mathrm{Nm}^{3}$ para un exceso de oxígeno base seca de $5.0 \mathrm{~mol} \%$. De los resultados mostrados en la Tabla 5, se observa que la concentración de partículas en base seca a $25^{\circ} \mathrm{C}$, $1 \mathrm{~atm}$ de presión y $5.0 \mathrm{~mol} \%$ de oxígeno de referencia $\left(\mathrm{C}_{\text {scnm }}\right)$ no rebasó el límite establecido en la normatividad mexicana, en ninguna de las mediciones realizadas.

De acuerdo con la tasa de emisión de PM10 de los tres $\mathrm{MCI}$, esta fue menor a la establecida por diseño $(10.9 \mathrm{~g} / \mathrm{s})$.

En el ámbito internacional, el Grupo Banco Mundial (GBM, 2007), estableció para centrales de combustión interna un nivel máximo de emisión de $50 \mathrm{mg} / \mathrm{Nm}^{3}$ de PST, considerando un exceso de oxígeno en base seca de $15.0 \mathrm{~mol} \%$. La concentración de partículas PST en base seca a $25^{\circ} \mathrm{C}, 1 \mathrm{~atm}$ y $15.0 \mathrm{~mol} \%$ de $\mathrm{O}_{2}$ de referencia $\left(\mathrm{C}_{\mathrm{scbm}}\right)$ de los tres motores de combustión interna (ver Tabla 5), fue superior a dicho límite e inclusive la concentración de PM10 del MCI-2 lo rebasó.

\section{Morfología de LAS PST y PM10}

En las Figuras 6, 7 y 8, se presenta la morfología de las partículas PST y PM10 colectadas en los MCI-1, MCI-2 y $\mathrm{MCI}-3$, respectivamente. En ellas se observa que las partículas obtenidas tanto en los filtros de PST como en los de PM10 poseen esencialmente la misma morfología: son sólidas, esféricas, con diámetros menores a 10 micras, con una estrecha distribución de tamaños y se encuentran aglomeradas, dejando solo una pequeña cantidad libre. Lo anterior confirma la similitud del proceso de combustión en los sistemas comparados. A este respecto, la gran cantidad de partículas pequeñas propició la formación de grumos o racimos y la concentración de PM10 fue de 61 al 80.0\% de las partículas capturadas en los MCI.

A diferencia de la PST emitidas por los MCI, la morfología de partículas producidas en un horno experimental de 0.35 MW que quema combustóleo son amorfas, porosas, de tamaños muy variados - generalmente mayores a 10 micras - y no presentan aglomeraciones, tal como se puede observar en la Figura 9 (Méndez, 2000)

\section{COMPOSICIÓN QUÍMICA ELEMENTAL DE LAS PST y PM10}

La composición elemental de las de PST y PM10 emitidas por las unidades de combustión interna se muestra en la Tabla 6. Dado el origen del combustible utilizado, los principales elementos que las componen son el carbono $(\mathrm{C})$, oxígeno $(\mathrm{O})$, vanadio $(\mathrm{V})$ y el azufre $(\mathrm{S})$. En la misma Tabla 6, se ha incluido la composición química de las partículas provenientes del horno experimental, en el cual, también se utilizó combustóleo como com-
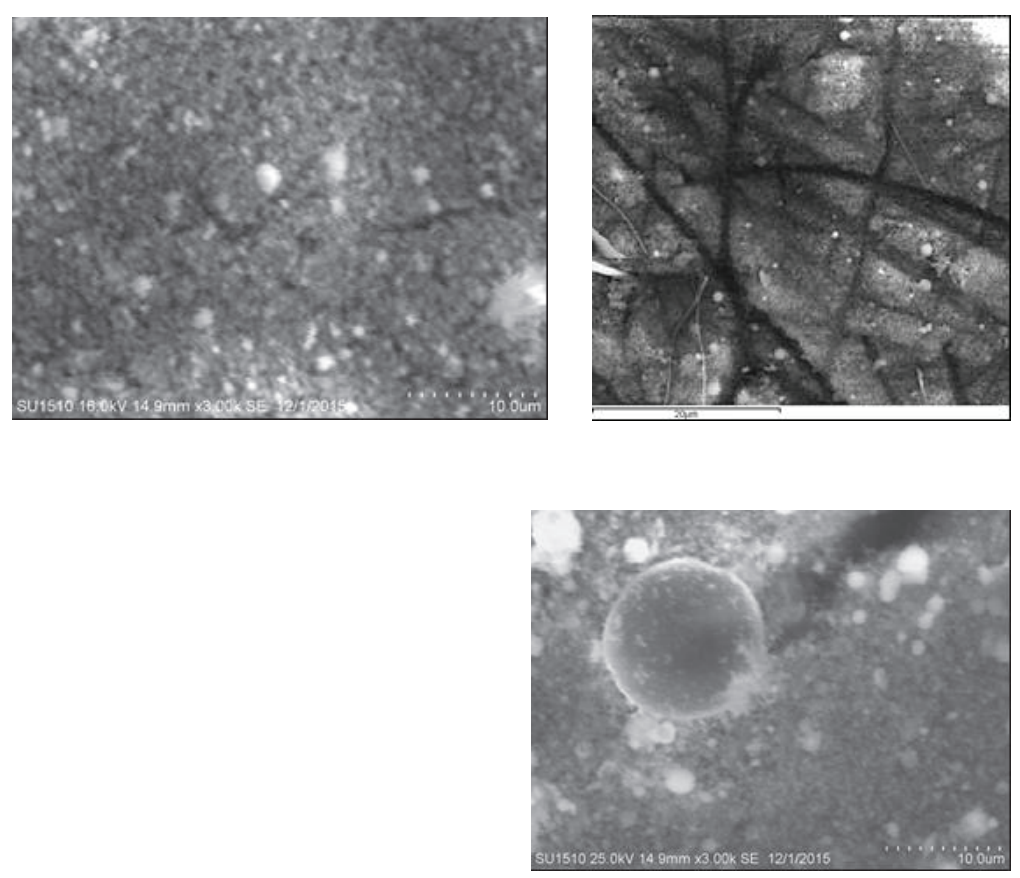

Figura 6. Micrografías de los filtros de PST y de PM10 del $\mathrm{MCl}-1$, respectivamente

Figura 7. Micrografía del filtro de PST del $\mathrm{MCl}-2$ 
a)

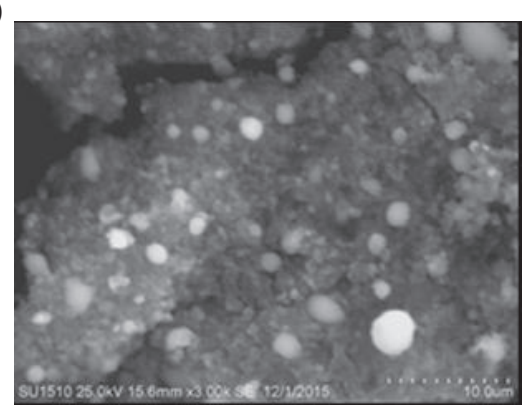

b)
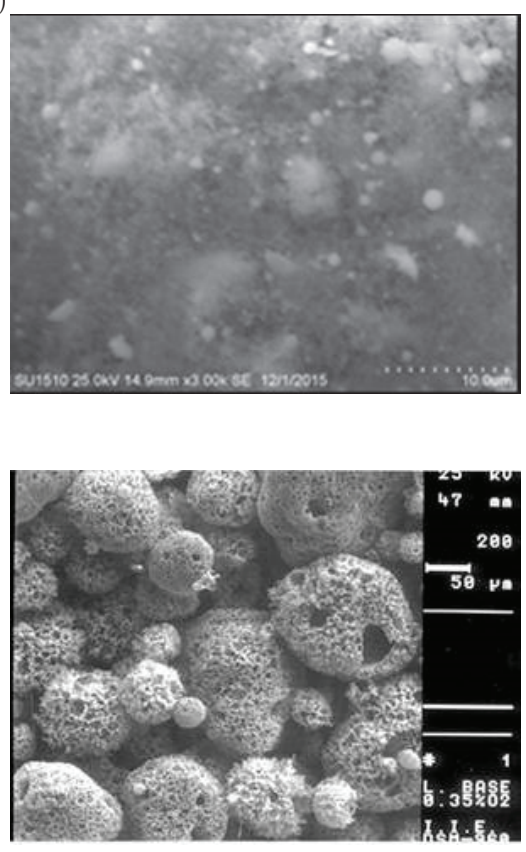

Figura 8. Micrografías de los filtros de a) PST y de b) PM10 del MCl-3

Figura 9. Micrografía de PST producidas en un horno experimental de 0.35 MW (Secretaría del Medio Ambiente y Recursos Naturales, 2011)

Tabla 6. Composición química elemental de partículas

\begin{tabular}{|c|c|c|c|c|c|}
\hline Elemento & $\begin{array}{c}\text { PST } \\
\text { MCI-1 } \\
\% \text { en peso } \\
\text { (85\% combustóleo/ } \\
15 \% \text { diésel) }\end{array}$ & $\begin{array}{c}\text { PST } \\
\text { MCI -2 } \\
\% \text { en peso } \\
\text { (Combustóleo) }\end{array}$ & $\begin{array}{c}\text { PST } \\
\text { MCI -3 } \\
\% \text { en peso } \\
\text { (Combustóleo) }\end{array}$ & $\begin{array}{c}\text { PM10 } \\
\text { MCI -3 } \\
\text { \% en peso } \\
\text { (Combustóleo) }\end{array}$ & $\begin{array}{l}\text { PST Horno experimental } \\
\% \text { en peso (Secretaría del } \\
\text { Medio Ambiente y Recursos } \\
\text { Naturales, 2011) } \\
\text { (Combustóleo) }\end{array}$ \\
\hline $\mathrm{C}_{(\mathrm{K})}$ & 33.78 & 19.62 & 26.90 & 21.54 & 64.70 \\
\hline $\mathrm{O}_{(\mathrm{K})}$ & 43.33 & 37.89 & 30.11 & 35.58 & ---- \\
\hline $\mathrm{Mg}_{(\mathrm{K})}$ & 0.28 & ---- & ---- & ---- & ---- \\
\hline $\mathrm{Al}_{(\mathrm{K})}$ & 0.96 & 0.56 & ---- & 0.55 & 0.49 \\
\hline $\mathrm{Na}_{(\mathrm{K})}$ & 0.44 & --- & --- & 0.43 & --- \\
\hline $\mathrm{Si}_{(\mathrm{K})}$ & 0.97 & 2.02 & 0.27 & 1.85 & 0.51 \\
\hline $\mathrm{P}_{(\mathrm{K})}$ & 0.27 & ---- & ---- & ---- & ---- \\
\hline$S_{(\mathrm{K})}$ & 4.44 & 5.02 & 2.83 & 4.12 & 18.80 \\
\hline $\mathrm{Ca}_{(\mathrm{K})}$ & 1.19 & 0.94 & 0.33 & 0.80 & 0.28 \\
\hline $\mathrm{V}_{(\mathrm{L})}$ & 13.30 & 33.41 & 39.26 & 34.67 & 11.48 \\
\hline $\mathrm{Fe}_{(\mathrm{K})}$ & 0.04 & 0.06 & 0.06 & 0.06 & 1.05 \\
\hline $\mathrm{Ni}_{(\mathrm{K})}$ & 0.16 & 0.48 & 0.24 & 0.39 & 2.60 \\
\hline $\mathrm{K}_{(\mathrm{K})}$ & & & & & 0.10 \\
\hline $\mathrm{Zn}_{(\mathrm{L})}$ & 0.85 & ---- & ---- & ---- & ---- \\
\hline Total & 100.01 & 100.00 & 100.00 & 99.99 & 100.01 \\
\hline $\begin{array}{l}\text { (K y L) Se refiere a las } \\
\text { capas de distribución } \\
\text { electrónica de los } \\
\text { átomos de cada } \\
\text { elemento }\end{array}$ & & & & & \\
\hline
\end{tabular}


bustible. Como se observa, la diferencia del número de elementos encontrados en las PST de la MCI-1 respecto a los elementos encontrados de los MCI-2 y 3, pueden explicarse por la diferencia de combustibles utilizados en ellos. Las PST de la MCI-1 presentan bajas concentraciones de magnesio $(\mathrm{Mg})$, sodio $(\mathrm{Na})$, fosforo $(\mathrm{P})$ y zinc ( $\mathrm{Zn}$ ), los cuales no se encuentran en las PST de los MCI-2 y 3. Los elementos encontrados tanto en las PST como en las PM10 de los MCI-2 y 3 tienen concentraciones menores de $\mathrm{C}$ y $\mathrm{O}$, pero casi tres veces la concentración de $\mathrm{V}$ obtenidas en las concentraciones de las PST del MCI-1.

Los contenidos de C, S, V y Ni en las PST y PM10 emitidas por los $\mathrm{MCI}$, forman componentes oxidantes en el ambiente con las mayores repercusiones en seres vivos. De ellos, el V es considerado uno de los más dañinos para el ser humano. A diferencia de las partículas mayores a 10 micrómetros, las PM10 penetran directamente al aparato respiratorio sin ser capturadas por sus mecanismos de limpieza (SEMARNAT, 2003). De acuerdo con su tamaño, una vez que estas partículas ingresan al tracto respiratorio, se acumulan en diferentes sitios dentro del aparato respiratorio. Las PM10 penetran hasta la zona traqueo bronquial, mientras que las PM2.5 pueden penetrar hasta los alvéolos pulmonares.

La evidencia acumulada hasta ahora indica que el aumento en las concentraciones de PM10 y PM2.5 se ha relacionado con el aumento de la mortalidad diaria y a largo plazo. A la inversa, cuando las concentraciones de partículas se reducen, la mortalidad también desciende, en el supuesto de que otros factores se mantengan sin cambios. Toda la población se ve afectada, pero la susceptibilidad a la contaminación puede variar con la salud o la edad (OMS, 2005).

\section{Conclusiones}

La concentración de PST en las tres MCI estuvo entre 151 y $190 \mathrm{mg} / \mathrm{Nm}^{3}$ misma que se encontró por debajo del límite establecido por la NOM-085-SEMARNAT-2011 (350 mg/ $\mathrm{Nm}^{3}$ ). Por su parte, la tasa de emisión de partículas PM10 fue inferior a la tasa de emisión especificada por diseño (10.9 g/s) para este tipo de máquinas de generación eléctrica. En relación con las recomendaciones del Banco Mundial, se tiene un nivel máximo de emisión de $50 \mathrm{mg} / \mathrm{Nm}^{3}$, que es 7 veces menor al límite máximo establecido por la NOM-085SEMARNAT-2011, por lo que, al comparar los resultados de PST de este estudio con el límite especificado por el Banco Mundial, se observó que estos fueron superiores, e incluso la concentración de PM10 del MCI-2 fue mayor.
Por otro lado, el porcentaje promedio de PM10 respecto a la emisión de PST en la MCI-1, fue de 61.8\%; mientras que, en las MCI-2 y 3 fue de $80 \%$. Los altos porcentajes de PM10 indican que la mayor cantidad de masa de las partículas generadas por las $\mathrm{MCI}$, pertenecen a la fracción de PM10, lo cual, se corroboró con el estudio de morfología.

La morfología observada muestra que la gran mayoría de las partículas producidas por las MCI fue menor a 10 micras, generado por la operación del sistema de inyección combustible, y de las condiciones físicas y operacionales de los equipos que participan en el proceso de combustión de las MCI.

\section{Perspectivas futuras}

Debido a que la composición elemental de las partículas reveló que existen elementos tóxicos como el vanadio y a que la técnica experimental empleada para la determinación de los mismos es semi-cuantitativa, se tiene contemplado que en trabajos futuros se adoptarán metodologías que permitan, tanto identificar como "cuantificar" la cantidad de cada uno de los elementos que constituyen las partículas.

Por las características de tamaño y forma de las partículas emitidas por las MCI, si las condiciones meteorológicas son propicias, estas pueden viajar en la corriente de la pluma y depositarse a grandes distancias, pudiendo alcanzar zonas urbanas, por lo que, se hace conveniente implementar tecnologías para el control de este tipo de partículas

\section{RefERENCIAS}

Environmental Protection Agency. Method 1-Sample velocity transverses for stationary sources, CFR Promulgated Test Methods (TM), 1994.

Environmental Protection Agency. Method 2-Determination of stack gas velocity and volumetric flow rate (Type S Pitot Tube), CFR Promulgated Test Methods (TM), 17 de septiembre de 2011.

Environmental Protection Agency. Method 3-Gas analysis for the determination of dry molecular weight, CFR Promulgated Test Methods (TM), 27 de octubre de 2009.

Environmental Protection Agency. Method 4-Determination of moisture content in stack gases, CFR Promulgated Test Methods (TM), 27 de octubre de 2009.

Environmental Protection Agency. Method 5-Determination of Particulate Emissions from Stationary Sources, CFR Promulgated Test Methods (TM), 27 de octubre de 2009. 
Environmental Protection Agency. Method 201A-Determination of PM10 and PM2.5 emissions from stationary sources (constant sampling rate procedure), 1 de noviembre de 2010.

Environmental Protection Agency. Sampling of ambient air for total Suspended Particulate Matter (SPM). Compendium of methods for the determination of inorganic compounds in ambient air, EPA/625/R-96/010A, 1996.

Espinoza J. Teoría y pruebas de combustión, Región de generación termoeléctrica Noreste, Comisión Federal de Electricidad, Capitulo IV, junio 1990.

Garrido A. y Camargo Y. Partículas respirables en el aire: generalidades y monitoreo en Latinoamérica. Revista INGE CUC, volumen 8 (número 1), 2012: 295-300.

Grupo Banco Mundial. Corporación Financiera Internacional (IFC). Guías sobre medio ambiente, salud y seguridad. Emisiones al aire y calidad del aire ambiente, abril de 2007.

Secretaría del Medio Ambiente y Recursos Naturales. Guía metodológica para la estimación de emisiones de PM2.5, Instituto Nacional de Ecología, 1ํㅡㄹ. ed., 2011.
Instituto Nacional de Ecología [en línea] [fecha de consulta: 26 de octubre de 2010]. Disponible en: http://www.ine.gob.mx/component/content/article/73-dica/523-calairecont-criterio

Méndez A.A. Microestructura de emulsiones agua en combustóleo pesado y su efecto en la combustión (tesis de maestría), Instituto Politécnico Nacional, noviembre de 2000, pp. 82-85.

Norma Oficial Mexicana NOM-085-SEMARNAT-2011, Diario Oficial de la Federación, 23 de abril de 2003.

Querol X. Congreso Nacional del Medio Ambiente 8, Cumbre del Desarrollo Sostenible, El Material Particulado Atmosférico. España, 2005, 28p. [en línea] Disponible en: http://www.conama8.org/modulodocumentos/documentos/AEs/AE9/AE9_ doc_XavierQuerol.pdf.

World Health Organization Europe. air quality guidelines, Global Update 2005, Particulate matter, ozone, nitrogen dioxide and sulfur dioxide, 2005. 


\section{Citación sugerida:}

\section{Citación estilo Chicago}

Martínez-Flores, Marco Antonio, Gustavo Adolfo Tamayo-Flores, Dulce Karime Barajas-Martínez, Nicasio Hernández-Flores, Ana Teresa Celada-Murillo, Alfonso Arias-Osorio, Elizabeth Cortes-López, Ana Laura Colin-Aguilar. Concentración, morfología y composición química de partículas PST y PM10, colectadas en la chimenea de tres motores de combustión interna de alta capacidad (42 MW) Ingeniería Investigación y Tecnología, XIX, 03 (2018): 291-303.

\section{Citación estilo ISO 690}

Martínez-Flores M.A., Tamayo-Flores G.A., Barajas-Martínez D.K., Hernandez-Flores N., Celada-Murillo A.T., Arias-Osorio A., CortesLópez E., Colin-Aguilar A.L. Concentración, morfología y composición química de partículas PST y PM10, colectadas en la chimenea de tres motores de combustión interna de alta capacidad (42 MW) Ingeniería Investigación y Tecnología, volumen XIX (número 3), julio-septiembre 2018: 291-303.

\section{Semblanzas de los autores}

Martínez-Flores Marco Antonio. Ingeniero Mecánico Electricista (1982) egresado de la FI, UNAM. Obtuvo el grado de Maestro en Ingeniería por la DEPFI de la UNAM (1988). En 1984 ingresó al INEEL en la especialidad de sistemas de combustión, fue jefe del Laboratorio de Combustión de la Gerencia de Procesos Térmicos, desde 2012 colabora en la División de Energías Alternas. Ha participado como jefe de proyecto y es coautor de dos patentes aceptadas y una más en proceso.

Tamayo-Flores Gustavo Adolfo. Ingeniero Mecánico egresado de la Universidad Michoacana de San Nicolás de Hidalgo en 1990. Obtuvo el grado de Maestro en Ingeniería Química en 2006 por la UAEM. En 1991 ingresó al INEEL en la especialidad de sistemas de combustión. Desde 2012 colabora en la División de Energías Alternas, ha participado como jefe de proyecto y es coautor de dos patentes aceptadas y una más en proceso.

Barajas-Martínez Dulce Karime. Ingeniera en Tecnología ambiental, egresada de la Universidad Politécnica de Estado de Morelos, 2016. En 2015 realizó su tesis de licenciatura en el INEEL, División Energías Alternas para la obtención de su título. Actualmente se encuentra estudiando la maestría en comercialización de conocimientos innovadores en el CIICAP de la UAEM.

Hernández-Flores Nicasio. Ingeniero Industrial egresado del Instituto Tecnológico de Orizaba en 2009. En 2012, obtuvo el grado de Maestro en Ingeniería Industrial por el Instituto Tecnológico de Orizaba. A partir del 2013 ingresó al INEEL en la División de Energías Alternas. Ha colaborado en diversos proyectos bajo contrato. Autor y coautor de diversos artículos en el área de ingeniería ambiental.

Celada-Murillo Ana Teresa. Doctorado en Ciencias por el CENIDET con especialidad en meteorología, micrometeorología, climatología urbana, modelación del transporte y dispersión de contaminantes atmosféricos con modelos propios y regulatorios aprobados por la EPA, evaluación de impacto de las emisiones de fuentes fijas sobre la calidad del aire y cómputo científico.

Arias-Osorio Alfonso. Ingeniero Ambiental egresado de la Universidad Autónoma Metropolitana. Actualmente es Jefe de Protección Ambiental de Proyectos Termoeléctricos de la Comisión Federal de Electricidad, área encargada de las gestiones ambientales, diseño y supervisión del equipamiento ambiental de centrales termoeléctricas.

Cortes-López Elizabeth. Ingeniero Químico por la Universidad Tecnológica de México con estudios de Posgrado en Calidad y Productividad. Jefa de la Disciplina de Calidad del Aire en el Departamento de Protección Ambiental de la Coordinación de Proyectos Termoeléctricos de la Comisión Federal de Electricidad.

Colín-Aguilar Ana Laura. Licenciada en Economía y Administración de Negocios por la Universidad del Sol en 2008 . En 2011 ingreso al INEEL en la División de Energías Alternas. En el 2015 se incorporó y colabora como investigadora en el grupo de Modelación Matemática, Micrometeorología y Contaminación Atmosférica. 
